\title{
Proactive inhibitory control of response as the default state of executive control
}

\author{
Marion Criaud ${ }^{1,2,3}$, Claire Wardak $^{1,2,3}$, Suliann Ben Hamed ${ }^{1,2,3}$, Bénédicte Ballanger ${ }^{1,2,3}$ and \\ Philippe Boulinguez ${ }^{1,2,3}$ *
}

\author{
1 Université de Lyon, Lyon, France \\ 2 Université Lyon 1, Villeurbanne, France \\ ${ }^{3}$ CNRS, UMR5229, Centre de Neuroscience Cognitive, Bron, France
}

Edited by:

Michael Dougherty, University of

Maryland at College Park, USA

Reviewed by:

Timothy Pleskac, Michigan State

University, USA

Fehmida Hussain, Middlesex

University Dubai, United Arab

Emirates

*Correspondence:

Philippe Boulinguez, Centre de

Neuroscience Cognitive, Institut des

Sciences Cognitives, 67 Boulevard

Pinel, 69675 Bron Cedex, France.

e-mail:philippe.boulinguez@isc.cnrs.fr
Refraining from reacting does not only involve reactive inhibitory mechanisms. It was recently found that inhibitory control also relies strongly on proactive mechanisms. However, since most available studies have focused on reactive stopping, little is known about how proactive inhibition of response is implemented. Two behavioral experiments were conducted to identify the temporal dynamics of this executive function. They manipulated respectively the time during which inhibitory control must be sustained until a stimulus occurs, and the time limit allowed to set up inhibition before a stimulus occurs. The results show that inhibitory control is not set up after but before instruction, and is not transient and sporadic but sustained across time. Consistent with our previous neuroimaging findings, these results suggest that proactive inhibition of response is the default mode of executive control. This implies that top-down control of sensorimotor reactivity would consist of a temporary release (up to several seconds), when appropriate (when the environment becomes predictable), of the default locking state. This conclusion is discussed with regard to current anatomo-functional models of inhibitory control, and to methodological features of studies of attention and sensorimotor control.

Keywords: executive control, response inhibition, go/nogo, alertness, warning, psychophysics, human

\section{INTRODUCTION}

Self-control relies on the ability to countermand inappropriate stimulus-driven response impulses. Several inhibitory mechanisms may contribute to the process of refraining from reacting. These are either phasic, reactive processes triggered by the external stimulus itself, or tonic, proactive processes driven by topdown control. While the former involve online control of ongoing responses, the latter entail anticipatory locking of movement triggering mechanisms.

Most available studies have focused on reactive stopping. However, recent developments in the cognitive neuroscience of inhibitory control have highlighted the probable role of proactive mechanisms in inhibitory control (see Aron, 2011 for review). Proactive inhibitory control of action might take various forms such as adjusting the level of motor readiness, gating movement triggering mechanisms non-selectively or, conversely, inhibiting a particular response tendency. Importantly, proactive and reactive mechanisms are probably not mutually exclusive but rather interact for efficient inhibitory control (Verbruggen and Logan, 2008; Ballanger, 2009; Ballanger et al., 2009; Chikazoe et al., 2009; Lo et al., 2009; Mazaheri et al., 2009; Boy et al., 2010c; Chen et al., 2010; Claffey et al., 2010; Duque et al., 2010; Karayanidis et al., 2010, 2011; Ullsperger and King, 2010; Zandbelt and Vink, 2010). Nevertheless, current models of inhibitory control are mainly based on studies that have investigated reactive mechanisms, and still very little is known about the recent notion of proactive inhibitory control.
The major obstacle for identifying proactive inhibitory control (Figure 1) is that it is almost always involved in stimulus-response paradigms (Jaffard et al., 2007). Indeed, when the experimental protocol introduces uncertainty about the next event to come (e.g., will it be a go or a nogo stimulus? a cue or a target? a square or a circle?), as is usually the case in mixed-block designs, subjects are forced to withhold automatic responses to any upcoming event in order to avoid erroneous responses. This creates a serious problem with respect to the meaning of the control conditions or baseline activities classically used to refer to the effects of interest. For example, the metabolic activity induced by proactive inhibitory control does not survive standard neuroimaging contrasts like nogo $>$ go or cue $>$ no-cue trials because the function is systematically involved in both conditions. Similarly, the persistent neuronal activity observed before a stimulus and pinpointing top-down inhibitory control in the absence of sensory input vanishes when a short epoch preceding the first event of interest is used as a baseline for event-related potentials in electroencephalographic or electrophysiological recordings. Thus, identifying proactive inhibitory control requires the use of an unbiased control condition performed in an independent block of trials in which response triggering mechanisms are not locked in advance (e.g., only go trials are presented apart from the other conditions of interest and are compared to go trials presented randomly in a mixed-design). Using appropriate control conditions and baselines in order to take into account the effect of proactive inhibitory control is central for the proper interpretation of the 


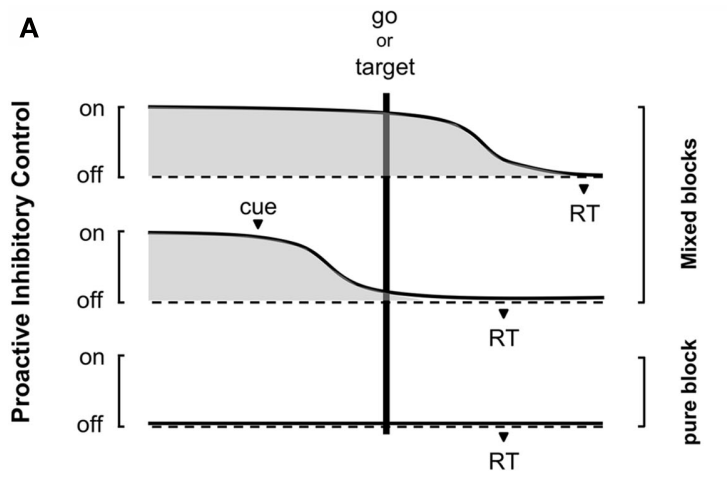

FIGURE 1 |The proactive inhibitory control model. (A) Hypothetical dynamics of proactive inhibitory control. When there is uncertainty about upcoming stimuli, as in standard mixed-block designs (e.g., go intermixed with nogo trials in go/nogo protocols - Figure2A, cue intermixed with no-cue trials in cue/target protocols - Figure $\mathbf{3}$ ), tonic response inhibition is required to prevent false alarms (automatic responses to nogo or to cue stimuli). This implies that inhibitory control cannot be lifted until the first stimulus has been identified (a go or a nogo, a cue or a target). As a consequence, proactive inhibitory control is maximum at target occurrence in go and no-cue trials (upper part), two conditions usually considered as regular control conditions in standard designs. Accordingly, the mechanism of action of a warning signal may simply consist in unbolting the gate before a target occurs (middle part). Importantly, no proactive inhibitory control is required in conditions in which only targets are presented (pure-blocks, lower part), providing an unbiased baseline rarely considered in psychophysical setups. (B) Behavioral correlates of proactive inhibitory control. Go trials in go/nogo protocols as well as no-cue trials in cue/target protocols show a dramatic increase in reaction times (RT) with respect to no-cue trials performed apart in pure-blocks. The former condition involves proactive inhibition while the latter does not. The model assumes that when a go signal is presented (or when a target occurs without being preceded by a warning cue), the stimulus needs first to be identified to allow the release of inhibitory control and, hence, movement initiation. If a warning cue is presented sufficiently in advance of the target (cue-target



onset asynchrony, CTOA $\geq 300 \mathrm{~ms}$ ), proactive inhibitory control has already been released at target occurrence and fast automatic responses to subsequent stimuli are generated in a similar way to no-cue trials performed apart in pure-blocks. (adapted from Jaffard et al., 2007). Yet, it could be argued that the RT change could be accounted for exclusively by (1) the addition of a visual discrimination process to the task in mixed-blocks, and (2) a linear rise to threshold model of motor preparation. Importantly, we previously tested and rejected this hypothesis. First, visual discrimination is a prerequisite in mixed designs, but ERP markers of inhibition are systematically locked to the process (e.g., Boulinguez et al., 2009). Second, electrophysiological measures of the activity of the muscles involved in response execution show that errors do not linearly increase as CTOA increases, as predicted by motor preparation effects. They are conversely composed of impulsive activations triggered by the first stimulus (Boulinguez et al., 2008; see also Sinclair and Hammond, 2008, 2009 for convincing evidence arising from studies using transcranial magnetic stimulation during foreperiods of warned RT privileging the inhibitory over the preparatory account). Finally, neuroimaging studies revealed that brain activity preceding stimulus presentation is coupled with changes in the motor brake circuitry (SMA, ventral anterior nucleus of the thalamus, primary motor cortex, putamen and inferior parietal lobule: Jaffard et al., 2007, 2008) and modulated by STN stimulation (Ballanger et al., 2009). In other words, proactive inhibitory control likely involves the anticipated suppression of the neuronal processes underlying movement initiation. neural mechanisms underlying the numerous cognitive functions usually tested with cue-target protocols (e.g., attention, decision making, executive control). Otherwise, this may lead to reversed interpretations of typical behavioral outcomes (Jaffard et al., 2007; Albares et al., 2011) or brain activations (Jaffard et al., 2007, 2008; Boulinguez et al., 2009). Taking into account the involvement of proactive inhibitory control in stimulus response experiments may help resolving long-lasting controversies about attention and cognitive control (Kok et al., 2006; Anderson, 2011).

The proactive inhibitory control model hypothesizes that subjects can switch from controlled inhibition of response (anticipated suppression of the neuronal processes underlying movement initiation) to automatic processing of sensorimotor information (unlocked state) depending on their expectations of upcoming events (see also Hikosaka and Isoda, 2010). Obviously, this control contributes to long-lasting task setting (the configuration of cognitive processes that is actively maintained for subsequent performance in the task; Sakai, 2008; Vallesi et al., 2009), but this switch can also be performed with short delays within the course of a trial. Indeed, the time required to release proactive inhibitory control was estimated at less than $300 \mathrm{~ms}$ (Jaffard et al.,
2007; Figure 1). But how proactive inhibitory control can be implemented remains elusive. It may be set up when the context becomes ambiguous or potentially conflicting. According to this view, executive control would consist of applying temporary inhibition when necessary. Conversely, proactive inhibition may be the default state of the executive system, meaning that topdown control of sensorimotor reactivity would consist of releasing temporarily proactive inhibitory control. The latter hypothesis is compatible with recent functional magnetic resonance imaging (fMRI) observations revealing large overlaps between the mysterious "Default Mode Network" of brain function and the structures involved in the tonic inhibition state (Jaffard et al., 2008), yet no direct behavioral support for this hypothesis is available.

Here we test these two hypotheses and assess the dynamics of proactive inhibitory control by asking two questions: First, can this control be sustained over long periods of time (several seconds) or is it transient? Second, how long does it take to set up proactive inhibitory control? Two behavioral experiments were conducted. They manipulated the time during which inhibitory control must be sustained until a stimulus occurs, and the time limit allowed to set up inhibition before a stimulus occurs. 


\section{EXPERIMENT 1}

Experiment 1 was adapted from classical go/nogo tasks. Subjects were asked to react to a go stimulus by means of a button press with the right thumb, and to withhold responses to an equiprobable nogo stimulus. Since we previously showed that proactive inhibitory control is largely involved in this kind of task (Jaffard et al., 2007), we also added a control condition in which it was not involved, i.e., go trials performed apart for which there is no need to refrain from reacting. In the go/nogo condition, we manipulated the time during which proactive inhibitory control had to be sustained by varying the delay between the beginning of a trial and the go or nogo stimulus presentation (Figure 2A).

\section{MATERIALS AND METHODS \\ Subjects}

Nineteen naive subjects (ages: 23-38, 11 females) with normal vision participated in the experiment. The experimental protocol was preapproved by the local ethical committee in Biomedical Research (comité de protection des personnes sud-est IV, No. CPP 11/025). All participants gave written informed consent.

\section{Apparatus and procedure}

The subjects were seated in a darkened room in front of a screen placed $50 \mathrm{~cm}$ from their eyes. Each subject's head was fixed using a chinrest to maintain the viewing distance and stabilize the head. Stimuli were presented and data were acquired using Presentation $^{\mathrm{TM}}$. A red cross $(0.4 \mathrm{~cm} \times 0.4 \mathrm{~cm})$ placed at the center of the screen and set at the subject's eye level served as a fixation point. Its appearance indicated the beginning of the trial and it lasted until the end of the trial. It could be replaced by a go stimulus (a $2.5-\mathrm{cm} \varnothing$ white circle centered on the screen, $2.9^{\circ}$ of visual angle), a nogo stimulus (a $2 \mathrm{~cm} \times 2 \mathrm{~cm}$ white " $\mathrm{X}$ " centered on the screen, $2.3^{\circ}$ of visual angle), or no stimulus at all (catch trials, $15 \%$ of trials). Pre-stimulus delays (time between the beginning of a trial and stimulus presentation, i.e., the time during which proactive inhibitory control had to be sustained) varied from 2 to $6 \mathrm{~s}$ by steps of $500 \mathrm{~ms}$. The inter-trial interval was fixed to $1 \mathrm{~s}$. Subjects were asked to react as fast as possible to go stimuli by pressing a button with the right thumb while maintaining their error rate (responses in absence of go stimulus, responses to nogo stimuli or missed go stimuli) below $10 \%$. They were informed about the evolution of their mean error rate after each error.

In order to introduce a control condition in which proactive inhibitory control was not involved, we added another kind of trial: a condition which did not require subjects to sustain proactive inhibitory control after the trial start and during the course of the pre-stimulus delay. In $35.7 \%$ of the trials, the central fixation point could turn white at the beginning of a trial, indicating that no nogo stimulus would be presented (only go/control - "white_cross" trials, $80 \%$ and catch trials, $20 \%$ ). This condition enabled subjects to react automatically to any upcoming event. After a training session of 50 trials, "white_cross" and "red_cross" conditions were presented randomly in mini-blocks of 20 trials. All in all, 36 trials were presented for each condition of interest: Go/nogo (go presented in the red_cross condition, i.e., with stimulus uncertainty), nogo (red_cross condition), and go/control (go presented in the white_cross condition, i.e., without uncertainty about the identity of the upcoming stimulus).

\section{Data analysis}

False alarm rate. A nine Pre-stimulus delay $(2 ; 2.5 ; 3 ; 3.5 ; 4 ; 4.5 ; 5$; $5.5 ; 6 \mathrm{~s}$ ) ANOVA with repeated-measures was applied to the False Alarm rate (number of responses to the nogo stimulus/number of nogo stimuli).

Reaction time (the time between a go stimulus presentation and the button press response). In order to avoid potential biases due to the classical lengthening of RT distribution's tail, we performed the statistical analysis of RT on log transformed data (neperian logarithm). A 2 Go (go/nogo vs. go/control) $\times 9$ Pre-stimulus delay $(2 ; 2.5 ; 3 ; 3.5 ; 4 ; 4.5 ; 5 ; 5.5 ; 6 \mathrm{~s})$ ANOVA with repeated-measures was applied. Post hoc comparisons were performed when necessary using Newman-Keuls tests.

\section{RESULTS}

\section{False alarms}

No significant effect was observed. The false alarm rate was low for all values of pre-stimulus delay (mean $0.158 \pm 0.026$ ).

\section{Reaction time}

Reaction time (RT) results are presented in Figure 2B. A main effect of Go $\left[F(1,18)=131, p<0.001 ; \eta^{2}=0.88\right]$ showed shorter RT to go stimuli in the go/control condition (RT: $293 \pm 43 \mathrm{~ms}$ ) than in the go/nogo condition (RT: $376 \pm 61 \mathrm{~ms}$ ). A main effect of Pre-stimulus delay was also observed $[F(8,144)=10.5, p<0.001$; $\left.\eta^{2}=0.37\right]$. Post hoc analyses revealed that RT decreased as a function of Pre-stimulus delay up to 3 s $(376 \pm 76$ vs. $359 \pm 66$ vs. $341 \pm 67$ for $2,2.5$, and $3 \mathrm{~s}$ delays respectively, $p s<0.05)$. No significant difference was observed between Pre-stimulus delay conditions above $3 \mathrm{~s}$. No significant interaction between go and Pre-stimulus delay factors was observed.

\section{DISCUSSION}

The suppression of actions that are inappropriate in a given context does not only rely on phasic, reactive inhibitory mechanisms triggered by a nogo signal. Knowing that inhibitory control of response may be required also involves anticipated adjustments. Indeed, in accordance with the results of the present experiment, the insertion of nogo signals in a series of trials delays response latency in go trials with respect to an appropriate go/control condition (Menon et al., 2001; Jaffard et al., 2007). Unfortunately, proper go/control conditions are not systematically or appropriately used or discussed in go/nogo tasks, and the mechanisms by which these anticipated modulations operate have long been ignored or neglected. Some studies have attributed proactive modulations of brain activity and related behavioral outcomes in inhibition tasks to varying levels of sustained attention (Mazaheri et al., 2009) or response readiness (van den Wildenberg et al., 2002). However, recent converging evidence indicates that foreknowledge of potentially conflicting upcoming events results in setting up a control set whereby inhibitory control of response is applied in advance (Jaffard et al., 2007, 2008; Boulinguez et al., 2008, 2009; Chikazoe et al., 2009; Lo et al., 2009; Boy et al., 2010c; Chen et al., 2010; 
A

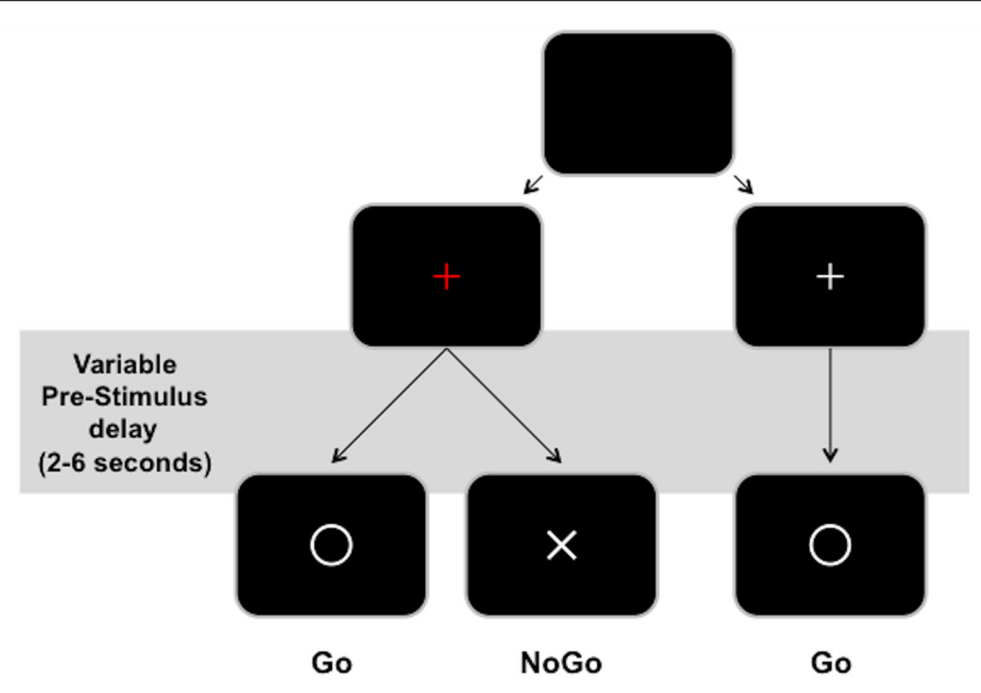

8

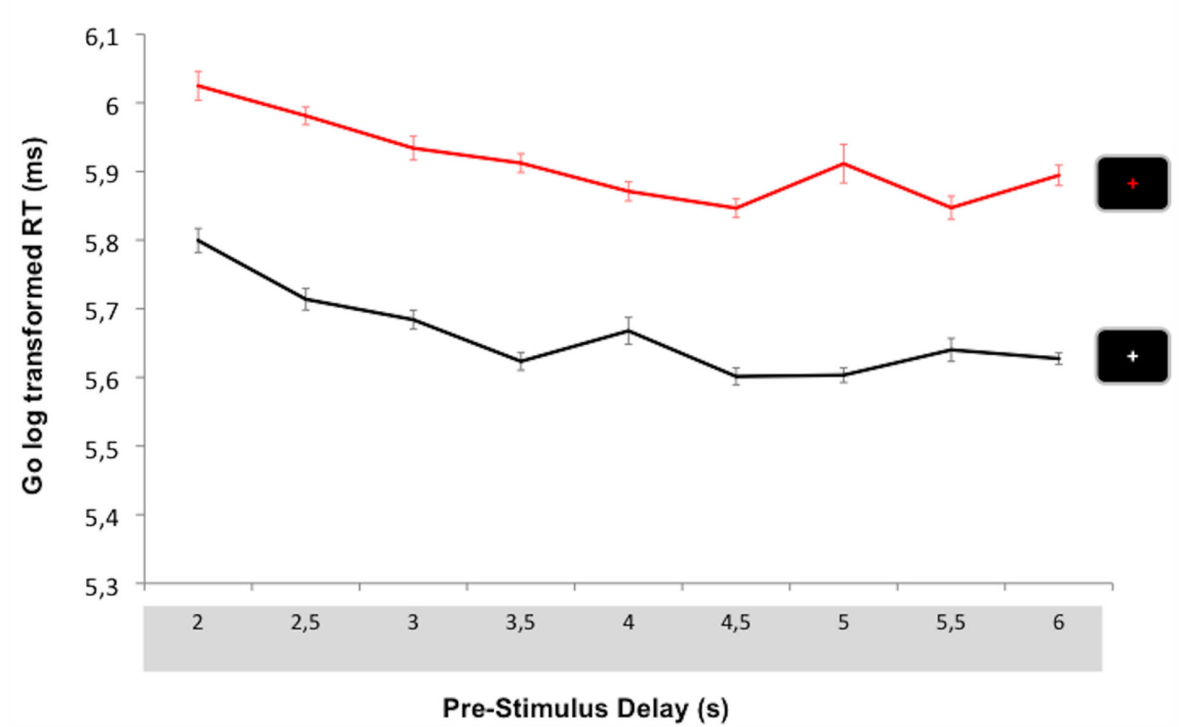

FIGURE 2 | (A) Overview of Experiment 1 (catch trials are not represented). Subjects are instructed to react to the presentation of a go signal $(\mathrm{O})$ by pressing a button as fast as possible. At the beginning of a trial, the central fixation point $(+)$ turns either red or white, indicating respectively that nogo stimuli $(X)$ can or cannot be presented. In the former condition, subjects must refrain from reacting in order to avoid responses to nogo stimuli. In the latter condition, subjects can react automatically to any upcoming target. (B) Mean reaction time to go/nogo (red line) and go/control (black line) stimuli plotted as a function of pre-stimulus delay. Vertical bars indicate standard error of the means.
Claffey et al., 2010; Stuphorn et al., 2010; Aron, 2011). Obviously, such an inhibitory state strongly affects performance in go/nogo trials because proactive response inhibition is a maximum at stimulus occurrence, in contrast with go/control trials in which no proactive inhibitory control applies since subjects do not need to withhold responses to upcoming stimuli. Accordingly, it is likely that the go/control vs. go/nogo RT difference is mostly due to the involvement of proactive inhibitory control. More precisely, this difference indexes the time required to release proactive inhibitory control of response after the stimulus has been identified as the go signal (Jaffard et al., 2007).

Based on this postulate, the results of Experiment 1 clearly suggest that proactive inhibitory control is not transient but can be sustained over several seconds (at least 6) since the go/control vs. go/nogo RT difference remains constant over increasing foreperiods ( $83 \mathrm{~ms}$, Figure $2 \mathbf{B}$ ). In particular, this main effect does not interact with the effect of foreperiod duration classically observed on RT when the distribution of pre-stimulus delays is uniform (Los and Van Den Heuvel, 2001). The observation that RT decreases as pre-stimulus delay increases (observed only from 2 to $3 \mathrm{~s}$ prestimulus delays in the present experiment) is usually attributed to the fact that non-specific preparation develops in accordance with the conditional probability of stimulus occurrence (the more time elapses, the greater the chance that a target will occur). Since this outcome is identical for the go/control and go/nogo conditions in our experiment, it can be asserted that proactive inhibitory 
control is not confounded with motor preparation. In other words, this observation supports the view that proactive inhibitory control of response does not only rely on the modulation of motor corticospinal excitability, but also involves independent breaking circuits acting on the command function for initiating a motor program (Jaffard et al., 2008).

While we now know that proactive inhibitory control can be sustained for relatively long periods of time (present data) and can be released when appropriate within extremely short delays (<300 ms, e.g., Jaffard et al., 2007, 2008; Boulinguez et al., 2009), a major issue remains regarding its dynamics: how is proactive inhibitory control implemented? Theoretically, two opposite hypotheses can be put forward: (1) Proactive inhibitory control is set up as soon as there is uncertainty about upcoming events, and is sustained until this uncertainty vanishes (the temporary set hypothesis). (2) Proactive inhibitory control is the default state of executive control, and can be actively released when an upcoming event becomes predictable (the default state hypothesis). These two hypotheses make specific predictions that are tested in Experiment 2.

\section{EXPERIMENT 2}

Experiment 2 (Figure 3) was adapted from the standard warned visual detection task. The original task allows the dynamics of proactive inhibitory control to be revealed because it separates in time the implementation of proactive inhibitory control, its release, and target presentation (Jaffard et al., 2007, 2008; Boulinguez et al., 2008, 2009), while the last two events occur simultaneously in go/nogo-like protocols. It was adapted in the sense that we used instruction signals as in Experiment 1 to inform subjects about the experimental condition (red and white crosses), rather than performing these conditions in two separate blocks of trials. Subjects were asked to react to a target, which might or might not be preceded by a warning cue, by means of a button press with the right thumb. In other words, they were implicitly asked to withhold responses to unpredictable cues. We manipulated the time limit to set up proactive inhibitory control by varying the delay between the beginning of a trial (instruction) and the stimulus presentation (cue or target, Figure 3 ). We focused on the period 0-2 s after the trial started, which was ignored in Experiment 1.

\section{MATERIALS AND METHODS \\ Subjects}

Twenty-five naive subjects (ages: 21-28, 17 females) with normal vision participated in the experiment. The experimental protocol was preapproved by the local ethical committee in Biomedical Research (comité de protection des personnes sud-est IV, No. CPP 11/025). All participants gave written informed consent.



FIGURE 3 | Overview of Experiment 2. Subjects are instructed to react to the presentation of the target $(X)$ by pressing a button as fast as possible. At the beginning of a trial, the central fixation point $(+)$ turns either red or white, indicating respectively that a warning cue (two peripheral squares) can or cannot be presented before target occurrence. In the former condition, subjects must refrain from reacting in order to avoid responses to the warning cue. In the latter condition, subjects can react automatically to any upcoming target. 


\section{Apparatus and procedure}

While keeping their gaze fixed on a central fixation point (a $0.4 \mathrm{~cm} \times 0.4 \mathrm{~cm}$ cross at the center of the screen), the subjects were asked to react as quickly as possible to a visual target that might or might not be preceded by a neutral warning signal. The neutral cue was composed of two $1.8 \mathrm{~cm} \times 1.8 \mathrm{~cm}$ squares $\left(2.06^{\circ}\right.$ of visual angle) located $6.9^{\circ}$ to the left and right of the fixation point (duration $50 \mathrm{~ms})$. The target was a $0.6 \mathrm{~cm} \times 0.5 \mathrm{~cm}$ " $X$ " $\left(0.69 \times 0.57^{\circ}\right.$ of visual angle) located either at $6.9^{\circ}$ to the left or right of the fixation point (duration $50 \mathrm{~ms}$ ).

As in Experiment 1, the central fixation point could be red or white when displayed at the beginning of a trial. A red cross indicated that a target could be preceded by a neutral warning signal $(75 \%)$ or not $(25 \%)$. A white cross indicated that no warning signal would be presented before a target (100\% targets). In other words, this control condition did not require subjects to sustain proactive inhibitory control during the course of the pre-stimulus delay. We manipulated the time allowed to implement proactive inhibitory control by varying the delay between the beginning of a trial (instruction) and stimulus presentation (cue or target; Figure 3). Pre-stimulus delays varied randomly from 250 to $2000 \mathrm{~ms}$ in steps of $250 \mathrm{~ms}$.

We also manipulated cue-target onset asynchrony (CTOA). Based on previous findings showing that the release of inhibitory control triggered by cue presentation takes less than $300 \mathrm{~ms}$ (Jaffard et al., 2007, 2008; Boulinguez et al., 2008, 2009), we used three CTOA levels that surround this value $(100,300$, and $500 \mathrm{~ms})$. Short CTOA $(100 \mathrm{~ms})$ is classically characterized by long RT because proactive inhibitory control is still engaged at target occurrence, whereas long CTOA $(300,500 \mathrm{~ms})$ is characterized by short RT because proactive inhibitory control has already been released at target occurrence. Importantly, RT values for long CTOA were always found to be identical to RT observed in a control condition in which only targets were presented and for which no proactive inhibitory control was required (Jaffard et al., 2007, 2008; Boulinguez et al., 2008, 2009). In other words, long CTOA also provide a reliable control condition.

After a training session of 50 trials, "white_cross" and "red_cross" conditions were presented randomly in 10 mini-blocks of 40 trials. All in all, 240 cue trials ( 80 for each CTOA condition), plus 80 red_cross_no_cue trials, plus 80 white_cross_no_cue trials were presented. For each one of these conditions, 10 trials were presented for each pre-stimulus delay.

\section{Data analysis}

False alarms. False alarms were defined as responses to the cue. There are easily detectable when the button press occurs before the target. However, there are less easy to detect when the delay between the cue and the target is short. In order to detect responses to cues given after the target was presented for short CTOA trials, we also applied a cut-off filter to RT $<150 \mathrm{~ms}$. These very short RT, outside the regular distribution, were considered as false alarms in cue target trials. An eight Pre-stimulus delay (250; 500; $750 ; 1000 ; 1250 ; 1500 ; 1750 ; 2000 \mathrm{~ms})$ ANOVA was applied to False Alarm rate (number of responses to the cue/number of cue trials).
Pre-processing of RT data. We first analyzed cue trials RT according to CTOA and Pre-stimulus delay. The goal of this preprocessing is to check that $\mathrm{CTOA} \geq 300 \mathrm{~ms}$ are long enough to allow subjects to release proactive inhibition, i.e., to insure that it is correct to consider long CTOA as a proper control condition. According to the model of proactive inhibitory control, this statement implies that longer RTs are observed for short $(100 \mathrm{~ms})$ than for long $(\geq 300 \mathrm{~ms}) \mathrm{CTOA}$, and that RT stabilizes after CTOA $300 \mathrm{~ms}$. A three CTOA (100, 300, $500 \mathrm{~ms})$ ANOVA confirmed that long CTOA (500 and $300 \mathrm{~ms}$ ) provides shorter RT than short CTOA $(100 \mathrm{~ms}$; respectively 252 and 254 vs. $307 \mathrm{~ms}$; $F(2,48)=147 ; p<0.001, p s<0.001$ for post hoc Newman-Keuls tests). Individual analyses ( $t$-tests) revealed that this effect was significant for each of our 25 subjects. Since no significant difference was observed between CTOA 500 and CTOA 300, we collapsed these two conditions, referred to as "long_CTOA" in the following sections, for the main data analyses.

Reaction time. Since switching from controlled inhibition to automatic sensorimotor reactivity may involve great interindividual variability with regard to switch timing (in addition to basic RT interindividual variability), we performed individual analyses to categorize each subject's behavior with respect to the specific predictions of our two hypotheses. Unpaired two-sample $t$-tests were used to compare the RTs of cue and no-cue conditions for each prestimulus delay. More precisely, we compared "red_cross_no_cue," "white_cross_no_cue," and "red_cross_long_CTOA" conditions. The false alarm rate (number of responses to cues/number of cues, all CTOA collapsed) for each pre-stimulus delay was assessed by means of unpaired two-sample $t$-tests.

\section{RATIONALE}

Figure 4 presents the specific predictions of the two opposite hypotheses.

If proactive inhibitory control is set up when uncertainty about upcoming events occurs (temporary set hypothesis), then, in our experimental design, set up is induced by the presentation of the red cross. According to this hypothesis (Figure 4, left side), the time required to implement proactive inhibitory control can easily be estimated on the basis of the analysis of cued trials performance (green lines). Indeed, when the cue appears before set up is completed, the false alarm rate (responses to cues) is expected to be maximum (with minimum RT to cues). When the cue appears after set up is completed, no false alarm is expected. This hypothesis suggests that the switch time from maximum to minimum false alarm rate reflects the time required to set up inhibitory control. No-cue trials in the uncertainty condition (red_cross, blue lines) provide specific predictions about the duration of this mechanism. Indeed, if the target appears within short delays, very short RTs are expected because proactive inhibitory control is not set yet. Conversely, if the target appears after a sufficient delay, long RTs are expected because inhibition is $\mathrm{ON}$ at target occurrence. The switch time from minimum to maximum RT is also supposed to index the time required to set up inhibitory control. Finally, no-cue trials in the control condition (white_cross, i.e., no need to set up proactive inhibitory control; black lines) predict short RT whatever the pre-stimulus delay. 
A

Inhibition OFF

Inhibition ON
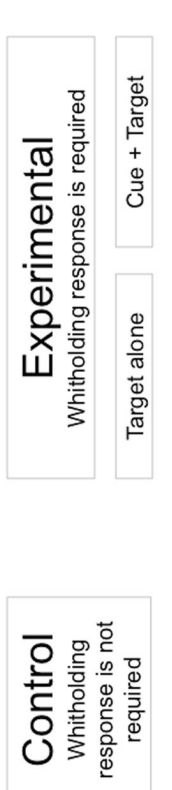

B

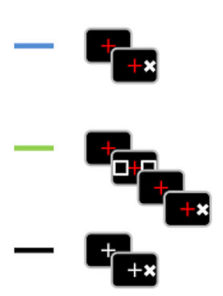

Hyp. \#1: Temporary Set


Time required to implement proactive inhibitory control

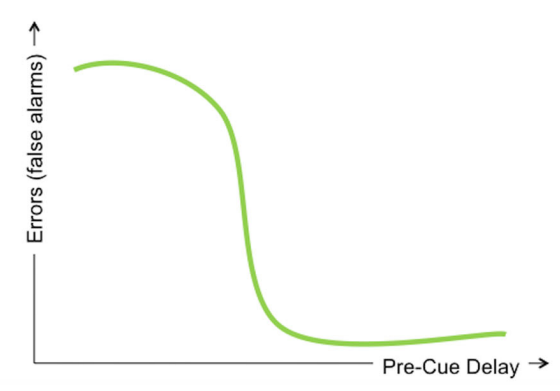

\section{Hyp. \#2: Default State}
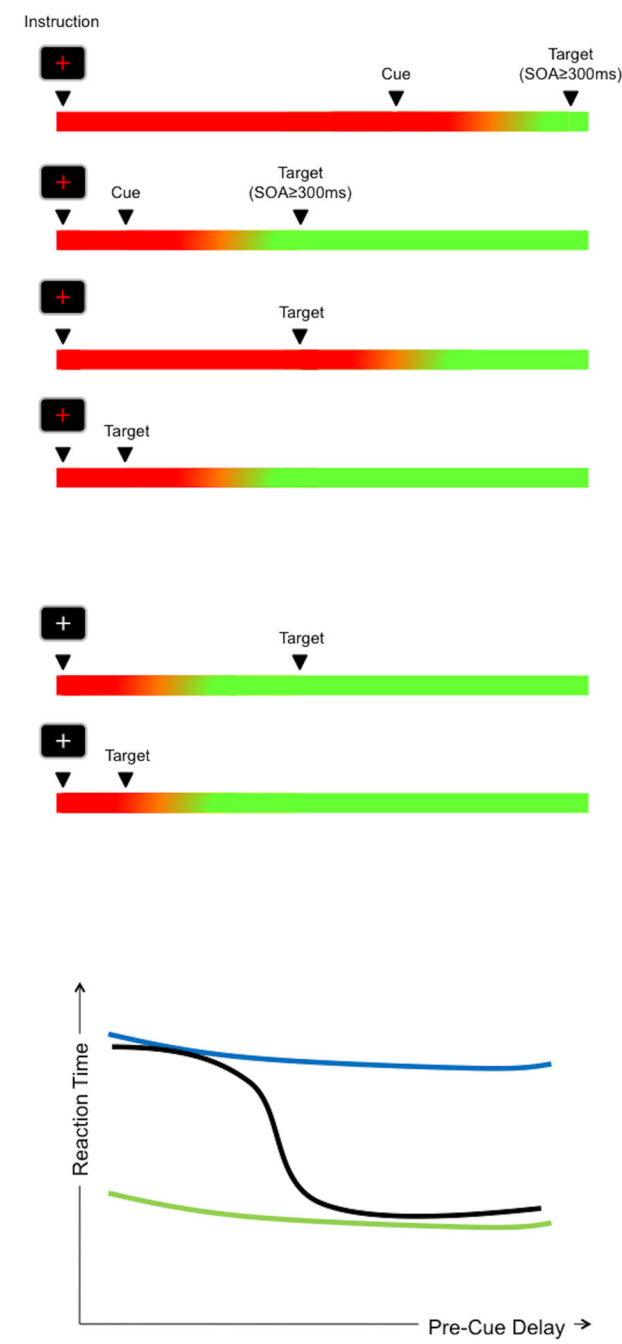

Time required to release proactive inhibitory control

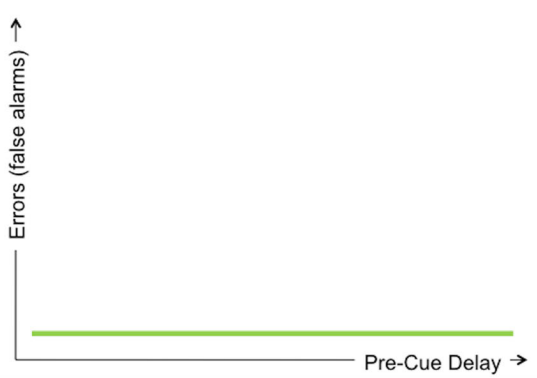

FIGURE 4 | Respective predictions of the temporary set and default state hypotheses of proactive inhibitory control. (A) The progress bars convey the state (ON or OFF) of proactive inhibitory control presupposed by each model at each moment in time, starting from the beginning of a trial, for each experimental condition. (B) Predictions regarding the evolution of both false alarms rate and RT according to pre-stimulus delay are presented (blue line: red_cross_no_cue; green line: red_cross_long_CTOA; black line: white_cross_no_cue). See text for details (see "Rationale"). 
The default state hypothesis makes opposite predictions. If proactive inhibitory control is the default state of executive control, this means it is already set at the trial start and must just be released after any event (white cross or warning cue) indicates that there is no more uncertainty about the identity of the next stimulus. According to this hypothesis (Figure 4, right side), the time required to release default inhibitory control can be estimated on the basis of the analysis of no-cue trials in the control condition (white_cross, i.e., no need to sustain proactive inhibitory control; black lines). Indeed, if the target appears too soon after instruction presentation, i.e., while inhibitory control is still $\mathrm{ON}$, then long RTs are expected. Conversely, if the target is presented after a sufficient delay, i.e., after inhibitory control has been released, short RTs are expected. This hypothesis suggests that the switch time from long to short RT reflects the time required to release the default state of inhibitory control. Since this control is already ON at the trial start, this hypothesis also predicts that the rate of false alarms will remain low whatever the duration of the pre-stimulus period for cued trials (green lines), as well as RT to targets with long CTOA. Finally, no-cue trials in the red_cross condition (blue lines) are expected to provide long RTs whatever the pre-stimulus delay because inhibitory control is always supposed to be $\mathrm{ON}$ at target occurrence.

To test these predictions, we performed individual statistical analyses. We first contrasted red_cross_no_cue with white_cross_no_cue conditions, assuming that inhibition is ON in the red_cross_no_cue condition when a significant difference is observed. If inhibition is $\mathrm{ON}$ in both conditions, no significant difference but long RT are expected. If inhibition is OFF in both conditions, no significant difference but short RT are expected. Then we contrasted white_cross_no_cue with red_cross_long_CTOA conditions, assuming that inhibition is $\mathrm{ON}$ in the white_cross_no_cue condition when a significant difference is observed, and OFF when there is no significant difference (see Figure 4; Table 1).

\section{RESULTS}

\section{False alarms}

No significant effect was observed. The false alarm rate was low for all values of pre-stimulus delay (mean $0.037 \pm 0.004)$.

\section{Reaction time}

Individual results are presented in Table 1 and Figure 5. Twentytwo of the 25 subjects showed similar patterns consistent with the predictions of Hypothesis \#2 (Figure 4).

Thus, we calculated a normalized RT in order to better illustrate the switch of the white_cross_no_cue condition from one control condition (inhibition $\mathrm{ON}$ ) to the other (inhibition OFF) as pre-stimulus delay elapses (Figures 4 and $5 \mathbf{A}$ ). White_cross_no_cue (black line) data were transformed with respect to statistical comparisons with red_cross_no_cue (blue line) and red_cross_long_CTOA (green line) control conditions for each pre-stimulus delay. When white_cross_no_cue is not different from red_cross_no_cue, RT is maximum. Accordingly, it was inferred that inhibition is $\mathrm{ON}$ at target occurrence and the value 1 was attributed to normalized white_cross_no_cue RT. Conversely, when white_cross_no_cue is not different from
red_cross_long_CTOA, RT is minimum. Thus, it was inferred that inhibition was OFF at target occurrence and the value 0 was attributed to normalized white_cross_no_cue RT. When significant differences were reported, corresponding normalized values were calculated as follows:

$$
\begin{aligned}
& \text { Normalized RT } \\
& \qquad=\frac{[\text { (red_cross_no_cue })-(\text { red_cross_long_CTOA })]}{[\text { (white_cross_no_cue })-(\text { red_cross_long_CTOA })]}
\end{aligned}
$$

Normalized RT data were then fitted by a logistic function (Figure 5A, right) which best represents the switch from one state to the other. A Chi-square was used to test the validity of this fit. Observed and theoretical distributions were not significantly different for any of the 22 subjects who showed patterns consistent with the predictions of Hypothesis \#2 ( $p$ s > 0.97; Table 1).

The time required to start releasing inhibitory control after a trial start in the white_cross_no_cue condition can be estimated as the time the fitted logistic function reports values $<1$. The time inhibitory control is fully released in the white_cross_no_cue condition can be estimated as the time the fitted logistic function reaches 0 . The difference between these two events represents the mean duration of the process in charge of releasing proactive inhibitory control after the uncertainty about the nature of the upcoming event has vanished. Figure $5 \mathrm{~B}$ shows that the timing of these events is variable across subjects (mean duration: $600 \pm 664 \mathrm{~ms}$ ).

Among the three remaining subjects, two (s8, s12) showed no difference between the red_cross_no_cue and white_cross_no_cue conditions. The other subject (s19) showed significant differences between the red_cross_no_cue and white_cross_no_cue conditions beginning $1750 \mathrm{~ms}$ after trial start. However, RT in the white_cross_no_cue condition remained longer than in the red_cross_long_CTOA control condition for all pre-stimulus delays. These two patterns do not fit all predictions of Hypothesis \#2, but are in total contradiction with the predictions of Hypothesis \#1.

\section{DISCUSSION}

Eighty-eight percent of the subjects revealed clear patterns of results fitting perfectly the predictions of the default state hypothesis (Figures 4 and 5A,B). Among the other subjects, two did not take into account the instruction to react freely to upcoming events (white_cross condition), or were unable to release inhibitory control in due time after trial start. Nevertheless, inhibitory control was also already set at trial start for these subjects, who were able to release it after the presentation of a warning cue in less than $300 \mathrm{~ms}$ like the other subjects. The other atypical subject seemed able to follow the instruction to release inhibitory control after the trial start (white_cross condition). However, his RT pattern suggests that this mechanism remained uncompleted $2 \mathrm{~s}$ after the trial start.

All together, these results suggest that (1) proactive inhibitory control is the default state of executive control because it is already set at the trial start for all subjects, (2) the dynamics of proactive inhibitory control of response is variable among subjects and is heterogeneous depending on the instructing stimulus: While 
Table 1 | Individual RT analyses of Experiment 2.

\begin{tabular}{|c|c|c|c|c|c|c|c|c|c|c|}
\hline Subject & Test & \multicolumn{8}{|c|}{ Pre-stimulus delay (ms) } & $\begin{array}{l}\text { Logistic fn fit } \\
\text { Chi-square }(p)\end{array}$ \\
\hline & \#2 & $*$ & * & $-(232)$ & - & - & - & - & - & \\
\hline & Norm. & 1 & 0.29 & 0 & 0 & 0 & 0 & 0 & 0 & 1 \\
\hline s2 & $\# 1$ & - & $-(457)$ & * & * & * & * & $*$ & * & \\
\hline \multirow[t]{3}{*}{ s3 } & $\# 1$ & $-\underline{(477)}$ & - & - & * & * & * & $*$ & * & \\
\hline & \#2 & $*$ & - & - & $-(\mathbf{2 4 9})$ & - & - & - & - & \\
\hline & Norm. & 1 & 0.54 & 0.20 & 0 & 0 & 0 & 0 & 0 & 1 \\
\hline \multirow[t]{2}{*}{ s4 } & $\# 1$ & $-\underline{(431)}$ & * & - & * & - & * & * & * & \\
\hline & \#2 & $*$ & * & - & - & - & $-(268)$ & - & - & \\
\hline s5 & Norm. & 0.55 & -0.55 & 0.59 & 0 & 0.24 & 0.25 & 0 & 0 & 0.99 \\
\hline \multirow[t]{3}{*}{ s6 } & $\# 1$ & $-(396)$ & $*$ & * & * & $*$ & $*$ & * & * & \\
\hline & \#2 & $*$ & * & $-(274)$ & - & - & - & - & - & \\
\hline & Norm. & 1 & 0.39 & 0 & 0 & 0 & 0 & 0 & 0 & 1 \\
\hline \multirow[t]{3}{*}{ s7 } & $\# 1$ & - & - & - & - & $-(301)$ & - & - & * & \\
\hline & \#2 & * & * & * & $*$ & * & - & - & $-(235)$ & \\
\hline & Norm. & 1 & 1 & 1 & 1 & 1 & 0.34 & 0.44 & 0 & 0.99 \\
\hline \multirow[t]{3}{*}{ s8 } & $\# 1$ & - & - & - & - & - & $-(299)$ & $*$ & * & \\
\hline & \#2 & * & * & * & * & * & * & $*$ & ${ }^{*}(239)$ & \\
\hline & Norm. & 1 & 1 & 1 & 1 & 1 & 1 & 0.63 & 1.1 & - \\
\hline s9 & $\# 1$ & - & $-(325)$ & - & - & * & * & $*$ & * & \\
\hline s11 & Norm. & 1 & 1 & 0.29 & 0 & 0 & 0 & 0 & 0 & 1 \\
\hline \multirow[t]{3}{*}{ s12 } & $\# 1$ & $-\underline{(450)}$ & - & - & - & - & - & - & - & \\
\hline & \#2 & ${ }^{*} \overline{(297)}$ & * & * & $*$ & * & * & * & * & \\
\hline & Norm. & 1 & 1 & 1 & 1 & 1 & 1 & 1 & 1 & - \\
\hline \multirow[t]{3}{*}{$\mathrm{s} 13$} & $\# 1$ & ${ }^{*}(504)$ & $*$ & * & $*$ & * & $*$ & $*$ & $*$ & \\
\hline & \#2 & $*$ & $-(242)$ & - & - & - & - & - & - & \\
\hline & Norm. & 0.33 & 0 & 0 & 0 & 0 & 0 & 0 & 0 & 1 \\
\hline \multirow[t]{3}{*}{ s14 } & $\# 1$ & $-(405)$ & * & * & * & * & * & * & * & \\
\hline & \#2 & * & $-(243)$ & - & - & - & - & - & - & \\
\hline & Norm. & 1 & 0 & 0 & 0 & 0 & 0 & 0 & 0 & 1 \\
\hline s15 & $\# 1$ & $-(372)$ & * & * & * & * & * & $*$ & * & \\
\hline & \#2 & - & $-(247)$ & - & - & - & - & - & - & \\
\hline & Norm. & 0.75 & 0 & 0 & 0 & 0 & 0 & 0 & 0 & 1 \\
\hline $\mathrm{s} 16$ & $\# 1$ & $-(414)$ & - & - & * & * & * & $*$ & $*$ & \\
\hline & \#2 & * & - & - & $-(234)$ & - & - & - & - & \\
\hline & Norm. & 1 & 0.46 & 0.20 & 0 & 0 & 0 & 0 & 0 & 0.99 \\
\hline s17 & $\# 1$ & $-(347)$ & $*$ & $*$ & * & * & * & * & * & \\
\hline
\end{tabular}


Table 1 | Continued

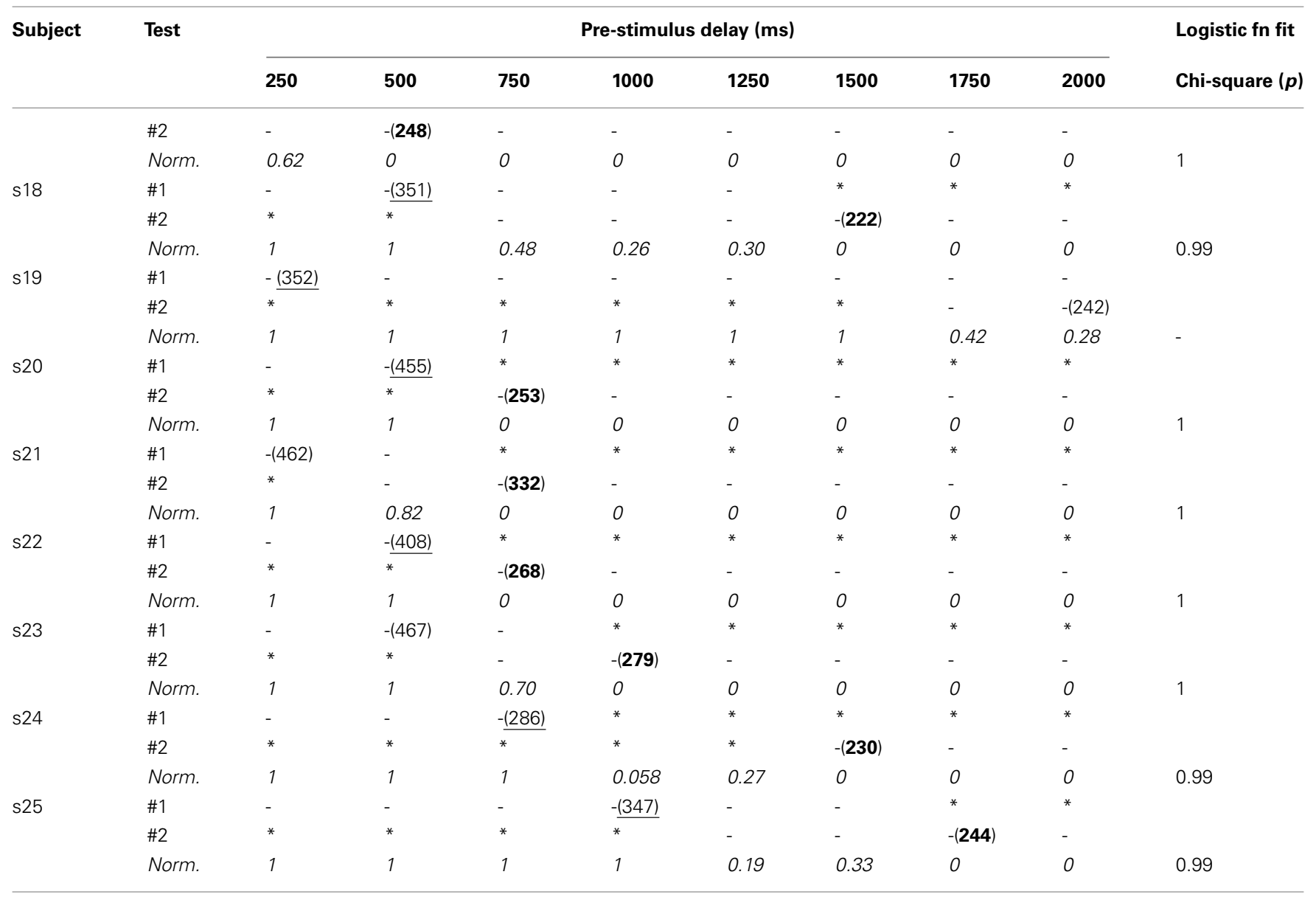

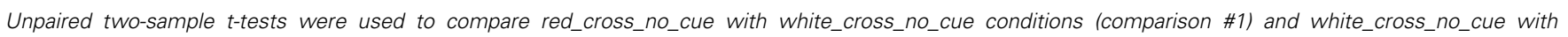

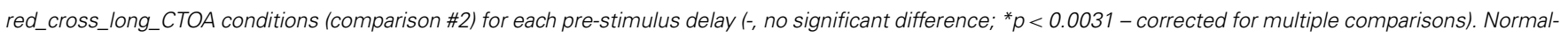

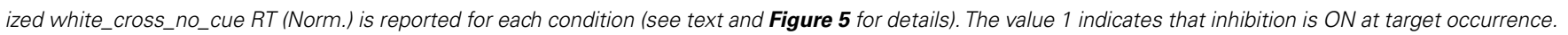




significantly reaches the OFF state. The p-values obtained when testing the validity of the logistic function fit (Chi-square) are reported in the last column.

an exogenous alerting cue allows a rapid $(<300 \mathrm{~ms})$ release of inhibitory control for all subjects, a symbolic instruction cue induces a longer and more variable switch to an automatic mode of sensorimotor processing ( $\geq 500 \mathrm{~ms}$ ).

\section{GENERAL DISCUSSION}

Proactive control may better account for efficient inhibition of inappropriate responses than reactive control (Aron, 2011). However, the mechanisms involved in the preparation to inhibit motor responses are still unclear. Brain activations (or deactivations) observed in the pre-stimulus period in tasks requiring subjects to refrain from reacting have been variously attributed to sustained attention (Coull et al., 1996; Coull, 1998; Mazaheri et al., 2009, 2011), motor corticospinal excitability reduction (Duque and Ivry, 2009; Sinclair and Hammond, 2009; Stinear et al., 2009), response threshold (Forstmann et al., 2008; Verbruggen and Logan, 2008), or increase in working memory load (Coull et al., 1996; McNab et al., 2008). Without refuting the direct or indirect roles these functions play in controlling motor outputs, we assume, based on recent findings, that the main neurocognitive mechanism involved in proactive inhibitory control is an active braking process that locks movement initiation processes in anticipation of stimulus occurrence (Jaffard et al., 2007, 2008 summarized in Figure 1; see also Brass and Haggard, 2007; Jahfari et al., 2009). However, how this control is set up is still unknown.

What is accepted is the fact that proactive inhibition is controlled according to the goals of the subject (Aron, 2011). The standard view implicitly suggests that this control is set up when the context becomes ambiguous or potentially conflicting. In other words, executive control would consist of generating a top-down signal to gate the neural mechanisms responsible for movement triggering as long as uncertainty remains. The results of the present experiment clearly contradict this view. Proactive inhibition of response is probably the default state of executive control. This means that applying control would conversely consist of generating a top-down signal that unlocks the neural mechanisms 

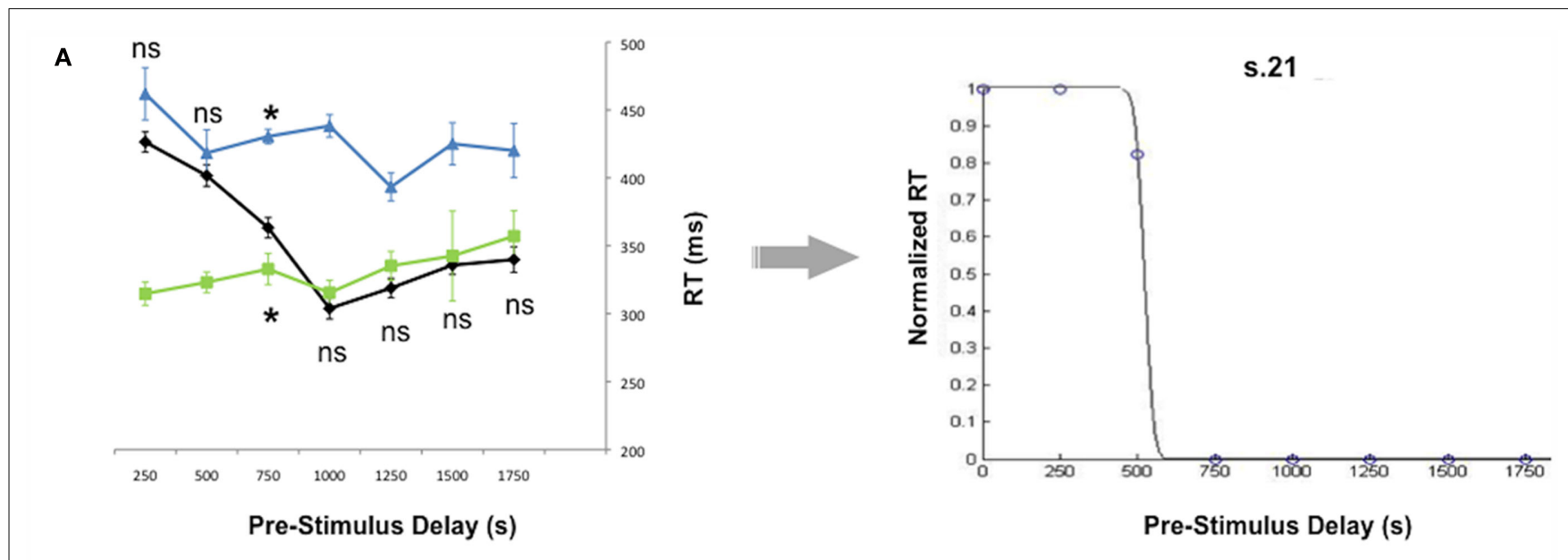

B

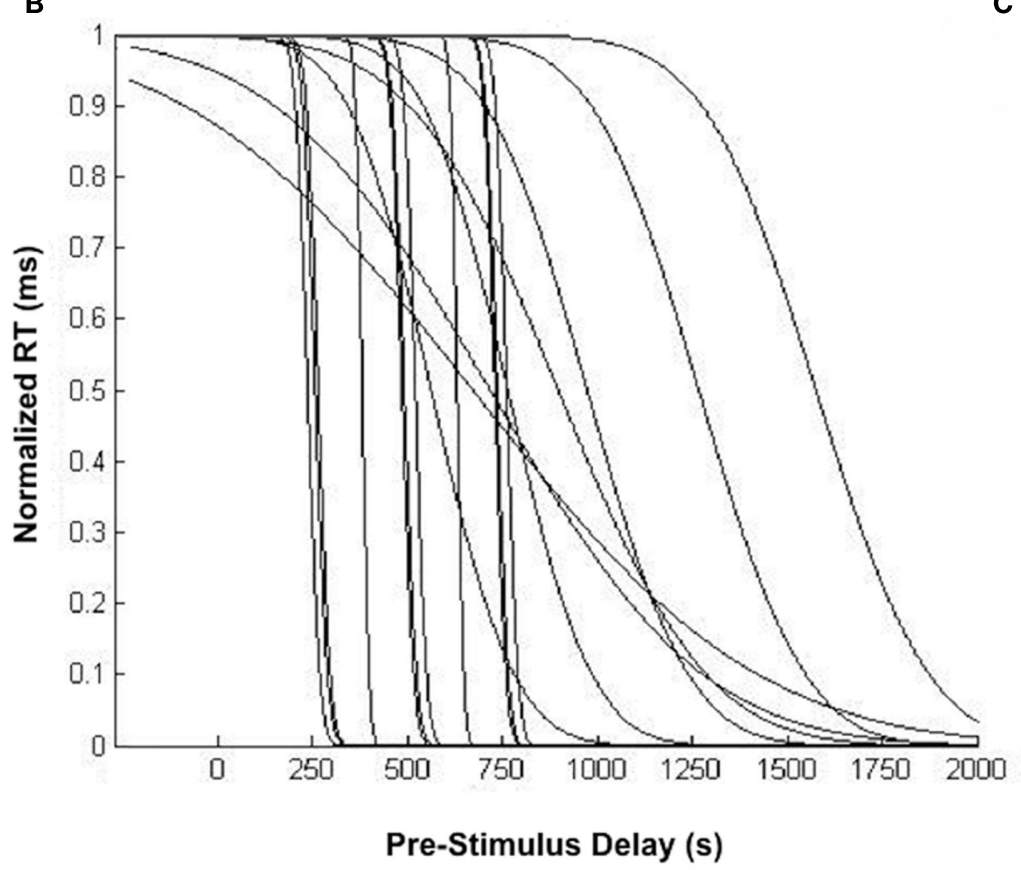

C

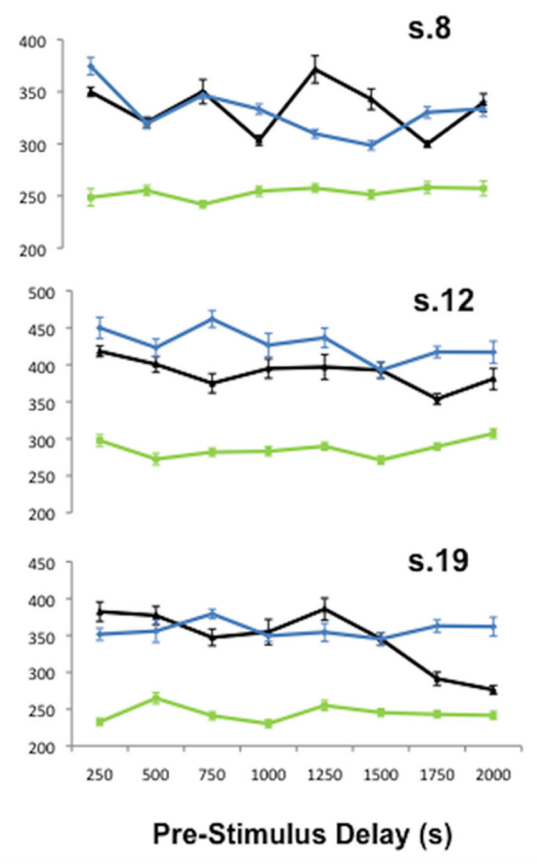

FIGURE 5 | Results of Experiment 2. (A) RT for a typical subject (left side).White_cross_no_cue RT normalized according to both red_cross_no_cue and red_cross_long_CTOA conditions for the same subject (right side). Data are fitted by a logistic function which best represents the switch from one state to another. (B) Fitted logistic functions for the 22 of 25 subjects whose data fit the predictions of the default state hypothesis of proactive inhibitory control. (C) RT for the three of the 25 subjects whose data did not match entirely the predictions of the default state hypothesis of proactive inhibitory control. Vertical bars indicate SE. responsible for movement triggering as soon as uncertainty about the next event had vanished. This release is not transient and probably does not act like a burst locked to the motor response, but can be sustained for several seconds. At first glance this mechanism seems counter-intuitive, but it is probably the most effective means of regulating voluntary vs. automatic modes of action control. While the ability to allow or to override automatic motor activations is a core feature of flexible and adaptive behavior, in everyday life, most of the time we must refrain from reacting to stimuli overflows. Indeed, all kinds of sensory stimulation may provide important sources of motor excitation that can trigger undesired movements (Endo et al., 1999; Tipper, 2001; Aron, 2007; Minelli et al., 2007; Boulinguez et al., 2008; Mele et al., 2008; Sumner and Husain, 2008). Only occasionally do we decide that acting or reacting may be appropriate. Accordingly, setting-up proactive inhibitory control each time the environment becomes potentially stimulating would be particularly inefficient, especially when the context is poorly predictable. In addition, from an ecological point of view, efficient control with reduced energy costs assumes that executive control consists of occasionally releasing, for short and appropriate periods of time, the default, effortless, mode of control that locks the neural mechanisms responsible for movement 
initiation. This mechanism is reminiscent of the neuroethological model of tonic inhibition/disinhibition that accounts for the control of motor programs both in vertebrate and invertebrate animals (Benjamin et al., 2010).

Obviously, the functional anatomy of this top-down control is still a hot matter of debate. Improving psychological models of proactive inhibitory control is required to feed future neuroimaging studies and contribute to a better understanding of the neural systems supporting this executive function. For instance, the knowledge that proactive inhibitory control is probably the default state of the executive system and that the top-down signal of interest may be locked to the "releasing" period should inspire future experimental settings aiming at distinguishing executive from attentional and sensorimotor processes (Jaffard et al., 2007, 2008). So far, it seems that proactive and reactive inhibition may engage partially overlapping brain networks, including especially the presupplementary motor area (preSMA), the right inferior frontal cortex, the subthalamic nucleus (STN), and the striatum (Vink et al., 2005; Ballanger et al., 2009; Jahfari et al., 2009; Zandbelt and Vink, 2010; Aron, 2011) as well as the supplementary motor area and the inferior parietal cortex (Menon et al., 2001; Rubia et al., 2001; Jaffard et al., 2008; Boy et al., 2010a,b; Chen et al., 2010; Swick et al., 2011; Wardak, 2011) with downstream effects on M1 excitability (Duque and Ivry, 2009; Sinclair and Hammond, 2009; Stinear et al., 2009; Claffey et al., 2010). In other words, the stopping network would be preactivated by preparing to inhibit (Aron, 2011). However, while highly probable, this provisional conclusion must be considered carefully because the respective effects of proactive and reactive mechanisms are difficult to disentangle one from the other with standard metabolic brain imaging methods (Jaffard et al., 2007, 2008; Boulinguez et al., 2008, 2009), and also because the physiological correlates of inhibition remain ambiguous (Lauritzen, 2001; Aron, 2007; Buzsáki et al., 2007). In addition, even when brain activity preceding stimulus processing is carefully isolated, it remains difficult to sort out proactive inhibition of response initiation from other functions involved in cognitive control like error monitoring (Rushworth and Taylor, 2007; Eichele et al., 2008) and task setting (Vallesi et al., 2009).

The conclusions of Experiment 2 are particularly reminiscent of our previous $\mathrm{fMRI}$ investigations suggesting that inhibitory control may be one of the functions of the "default mode of brain function" (Jaffard et al., 2007, 2008). Indeed, the mPFC (together with the precuneus/posterior cingulate and inferior parietal cortex also identified in the above mentioned studies, see Hagmann et al., 2008, for supporting anatomo-functional description) is characterized by important "intrinsic" activity during resting states (Raichle et al., 2001; Raichle, 2006; Raichle and Snyder, 2007). It is noteworthy that this activity is especially evidenced in experiments in which subjects are explicitly instructed to "refrain from moving and reacting" in passive viewing conditions (Mazoyer et al., 2001). The meaning of these resting state activations is still controversial. By providing behavioral evidence that proactive inhibition of response is the default mode of executive control, the present data strongly support our former suggestion that the activity at rest may be partly due to an active and sustained process consisting of locking movement initiation mechanisms. Nevertheless, understanding the intrinsic brain activity preceding stimulation is still a challenge of critical importance, and a large amount of work is still needed to understand precisely how proactive inhibitory control works. We hope that the findings described in this paper will suggest theoretical and methodological lines of inquiry that will contribute to achieving this goal.

\section{ACKNOWLEDGMENTS}

This work was supported by a grant ANR (MNPS-039-01) to Philippe Boulinguez. The authors thank the Centre Hospitalier St Jean de Dieu for promoting this research program.

\section{REFERENCES}

Albares, M., Criaud, M., Wardak, C., Nguyen, S. C. T., Ben Hamed, S., and Boulinguez, P. (2011). Attention to baseline: does orienting visuospatial attention really facilitate target detection? J. Neurophysiol. 106, 809-816.

Anderson, B. (2011). There is no such thing as attention. Front. Psychol. 2:246. doi:10.3389/fpsyg.2011.00246

Aron, A. R. (2007). The neural basis of inhibition in cognitive control. Neuroscientist 13, 214-228.

Aron, A. R. (2011). From reactive to proactive and selective control: developing a richer model for stopping inappropriate responses. Biol. Psychiatry 69, e55-e68.

Ballanger, B. (2009). Top-down control of saccades as part of a generalized model of proactive inhibitory control. J. Neurophysiol. 102, 2578-2580.
Ballanger, B., van Eimeren, T., Moro, E., Lozano, A. M., Hamani, C., Boulinguez, P., Pellecchia, G., Houle, S., Poon, Y. Y., Lang, A. E., and Strafella, A. (2009). Stimulation of the subthalamic nucleus and impulsivity: release your horses. Ann. Neurol. 66, 817-824.

Benjamin, P. R., Staras, K., and Kemenes, G. (2010). What roles do tonic inhibition and disinhibition play in the control of motor programs? Front. Behav. Neurosci. 4:30. doi:10.3389/fnbeh.2010.00030

Boulinguez, P., Ballanger, B., Granjon, L., and Benraiss, A. (2009). The paradoxical effect of warning on reaction time: demonstrating proactive response inhibition with eventrelated potentials. Clin. Neurophysiol. 120, 730-737.

Boulinguez, P., Jaffard, M., Granjon, L., and Benraiss, A. (2008). Warning signals induce automatic EMG activations and proactive volitional inhibition: evidence from analysis of error distribution in simple RT. J. Neurophysiol. 99, 1572-1578.

Boy, F., Evans, J., Edden, R. A. E., Husain, M., Singh, K. D, Husain, M., and Sumner, P. (2010a). Individual differences in subconscious motor control predicted by GABA concentration in SMA. Curr. Biol.20, 1779-1785.

Boy, F., Husain, M., Singh, K. D., and Sumner, P. (2010b). Supplementary motor area activations in unconscious inhibition of voluntary action. Exp. Brain Res. 206, 441-448.

Boy, F., Husain, M., and Sumner, P. (2010c). Automatic unconscious inhibition separates two forms of top-down control. Proc. Natl. Acad. Sci. U.S.A. 107, 11134-11139.

Brass, M., and Haggard, P. (2007). To do or not to do: the neural signature of self-control. J. Neurosci. 27, 9141-9145.

Buzsáki, G., Kaila, K., and Raichle, M. (2007). Inhibition and brain work. Neuron 56, 771-783.

Chen, X., Scangos, K. W., and Stuphorn, V. (2010). Supplementary motor area exerts proactive and reactive control of arm movements. J. Neurosci. 30, 14657-14675.

Chikazoe, J., Jimura, K., Hirose, S., Yamashita, K., Miyashita, Y., and Konishi, S. (2009). Preparation to inhibit a response complements response inhibition during performance of a stop-signal task. J. Neurosci. 29, 15870-15877.

Claffey, M. P., Sheldon, S., Stinear, C. M., Verbruggen, F., and Aron, A. R. (2010). Having a goal to stop action is associated with advance control of specific motor representations. Neuropsychologia 48, $541-548$. 
Coull, J. T. (1998). Neural correlates of attention and arousal: insights from electrophysiology, functional neuroimaging and psychopharmacology. Prog. Neurobiol. 55, 343-361.

Coull, J. T., Frith, C. D., Frackowiak, R. S. J., and Grasby, P. M. (1996). A fronto-parietal network for rapid visual information processing: a PET study of sustained attention and working memory. Neuropsychologia 34, 1085-1095.

Duque, J., and Ivry, R. B. (2009). Role of corticospinal suppression during motor preparation. Cereb. Cortex 19, 2013-2024.

Duque, J., Lew, D., Mazzocchio, R., Olivier, E., and Ivry, R. B. (2010). Evidence for two concurrent inhibitory mechanisms during response preparation. J. Neurosci. 30, 3793-3802.

Eichele, T., Debener, S., Calhoun, V. D., Specht, K., Engel, A. K., Hugdahl, K., von Cramon, D. Y., and Ullsperger, M. (2008). Prediction of human errors by maladaptive changes in event-related brain networks. Proc. Natl. Acad. Sci. U.S.A. 105, 6173-6178.

Endo, H., Kizuka, T., Masuda, T., and Takeda, T. (1999). Automatic activation in the human primary motor cortex synchronized with movement preparation. Brain Res. Cogn. Brain Res. 8, 229-239.

Forstmann, B. U., Dutilh, G., Brown, S., Neumann, J., von Cramon, D. Y., Ridderinkhof, K. R., and Wagenmakers, E.-J. (2008). Striatum and pre-SMA facilitate decision-making under time pressure. Proc. Natl. Acad. Sci. U.S.A. 105, 17538-17542.

Hagmann, P., Cammoun, L., Gigandet, X., Meuli, R., Honey, C. J., Wedeen, V. J., and Sporns, O. (2008). Mapping the structural core of human cerebral cortex. PLoS Biol. 6, e159. doi:10.1371/journal.pbio.0060159

Hikosaka, O., and Isoda, M. (2010). Switching from automatic to controlled behavior: cortico-basal ganglia mechanisms. Trends Cogn. Sci. (Regul. Ed.) 14, 154-161.

Jaffard, M., Benraiss, A., Longcamp, M., Velay, J.-L., and Boulinguez, P. (2007). Cueing method biases in visual detection studies. Brain Res. 1179, 106-118.

Jaffard, M., Longcamp, M., Velay, J.-L., Anton, J.-L., Roth, M., Nazarian, B., and Boulinguez, P. (2008). Proactive inhibitory control of movement assessed by event-related fMRI. Neuroimage 42, 1196-1206.

Jahfari, S., Stinear, C. M., Claffey, M., Verbruggen, F., and Aron, A. R. (2009). Responding with restraint: what are the neurocognitive mechanisms? J. Cogn. Neurosci. 22, 1479-1492.
Karayanidis, F., Jamadar, S., Ruge, H., Phillips, N., Heathcote, A., and Forstmann, B. U. (2010). Advance preparation in task-switching: converging evidence from behavioral, brain activation, and model-based approaches. Front. Psychol. 1:25. doi:10.3389/fpsyg.2010.00025

Karayanidis, F., Whitson, L. R., Heathcote, A., and Michie, P. T. (2011). Variability in proactive and reactive cognitive control processes across the adult lifespan. Front. Psychol. 2:318. doi:10.3389/fpsyg.2011.00318

Kok, A., Ridderinkhof, K. R., and Ullsperger, M. (2006). The control of attention and actions: current research and future developments. Brain Res. 1105, 1-6.

Lauritzen, M. (2001). Relationship of spikes, synaptic activity, and local changes of cerebral blood flow. J. Cereb. Blood Flow Metab. 21, 1367-1383.

Lo, C.-C., Boucher, L., Paré, M., Schall, J. D., and Wang, X.-J. (2009). Proactive inhibitory control and attractor dynamics in countermanding action: a spiking neural circuit model. J. Neurosci. 29, 9059-9071.

Los, S. A., and Van Den Heuvel, C. E. (2001). Intentional and unintentional contributions of nonspecific preparation during reaction time foreperiods. J. Exp. Psychol. Hum. Percept. Perform. 27, 370.

Mazaheri, A., DiQuattro, N. E., Bengson, J., and Geng, J. J. (2011). Pre-stimulus activity predicts the winner of topdown vs. bottom-up attentional selection. PLOS ONE 6, e16243. doi:10.1371/journal.pone.0016243

Mazaheri, A., Nieuwenhuis, I. L. C., van Dijk, H., and Jensen, O. (2009). Prestimulus alpha and mu activity predicts failure to inhibit motor responses. Hum. Brain Mapp. 30, 1791-1800.

Mazoyer, B., Zago, L., Mellet, E., Bricogne, S., Etard, O., Houdé, O. Crivello, F., Joliot, M., Petit, L., and Tzourio-Mazoyer, N. (2001). Cortical networks for working memory and executive functions sustain the conscious resting state in man. Brain Res. Bull. 54, 287-298.

McNab, F., Leroux, G., Strand, F., Thorell, L., Bergman, S., and Klingberg, T. (2008). Common and unique components of inhibition and working memory: an fMRI, within-subjects investigation. $\mathrm{Neu}$ ropsychologia 46, 2668-2682.

Mele, S., Savazzi, S., Marzi, C. A., and Berlucchi, G. (2008). Reaction time inhibition from subliminal cues: is it related to inhibition of return? Neuropsychologia 46, 810-819.
Menon, V., Adleman, N. E., White, C. D. Glover, G. H., and Reiss, A. L. (2001). Error-related brain activation during a go/nogo response inhibition task. Hum. Brain Mapp. 12, 131-143. Minelli, A., Marzi, C. A., and Girelli, M. (2007). Lateralized readiness potential elicited by undetected visual stimuli. Exp. Brain Res. 179, 683-690.

Raichle, M. E. (2006). The brain's dark energy. Sci. Am. 314, 1249.

Raichle, M. E., MacLeod, A. M., Snyder, A. Z., Powers, W. J., Gusnard, D. A. and Shulman, G. L. (2001). A default mode of brain function. Proc. Natl. Acad. Sci. U.S.A. 98, 676-682.

Raichle, M. E., and Snyder, A. Z. (2007). A default mode of brain function: a brief history of an evolving idea. Neuroimage 37, 1083-1090.

Rubia, K., Russell, T., Overmeyer, S. Brammer, M. J., Bullmore, E. T. Sharma, T., Simmons, A., Williams, S. C. R., Giampietro, V., Andrew, C. M., and Taylor, E. (2001). Mapping motor inhibition: conjunctive brain activations across different versions of go/no-go and stop tasks. Neuroimage 13, 250-261.

Rushworth, M. F. S., and Taylor, P. C. J. (2007). A paradoxical role for inhibition in initiation. Neuron 54 669-670.

Sakai, K. (2008). Task set and prefrontal cortex. Annu. Rev. Neurosci. 31, 219-245.

Sinclair, C., and Hammond, G. R. (2008). Reduced intracortical inhibition during the foreperiod of a warned reaction time task. Exp. Brain Res. 186, 385-392.

Sinclair, C., and Hammond, G. R (2009). Excitatory and inhibitory processes in primary motor cortex during the foreperiod of a warned reaction time task are unrelated to response expectancy. Exp. Brain Res. 194, 103-113.

Stinear, C. M., Coxon, J. P., and Byblow, W. D. (2009). Primary motor cortex and movement prevention: where Stop meets Go. Neurosci. Biobehavi. Rev. 33, 662-673.

Stuphorn, V., Brown, J. W., and Schall, J. D. (2010). Role of supplementary eye field in saccade initiation: executive, not direct, control. J. Neurophysiol. 103, 801-816.

Sumner, P., and Husain, M. (2008). At the edge of consciousness: automatic motor activation and voluntary control. Neuroscientist 14 474-486.

Swick, D., Ashley, V., and Turken, U. (2011). Are the neural correlates of stopping and not going identical? Quantitative meta-analysis of two response inhibition tasks. Neuroimage 56, 1655-1665.
Tipper, S. P. (2001). Does negative priming reflect inhibitory mechanisms? A review and integration of conflicting views. Q. J. Exp. Psychol. A 54, 321-343.

Ullsperger, M., and King, J. A. (2010). Proactive and reactive recruitment of cognitive control: comment on Hikosaka and Isoda. Trends Cogn. Sci. (Regul. Ed.) 14, 191-192.

Vallesi, A., McIntosh, A. R., Alexander, M. P., and Stuss, D. T. (2009). fMRI evidence of a functional network setting the criteria for withholding a response. Neuroimage 45, 537-548.

van den Wildenberg, W. P., van der Molen, M. W., and Logan, G. D. (2002). Reduced response readiness delays stop signal inhibition. Acta Psychol. (Amst.) 111, 155-169.

Verbruggen, F., and Logan, G. D. (2008). Automatic and controlled response inhibition: associative learning in the go/no-go and stop-signal paradigms. J. Exp. Psychol. Gen. 137, 649-672.

Vink, M., Kahn, R. S., Raemaekers, M., van den Heuvel, M., Boersma, M., and Ramsey, N. F. (2005). Function of striatum beyond inhibition and execution of motor responses. Hum. Brain Mapp. 25, 336-344.

Wardak, C. (2011). The role of the supplementary motor area in inhibitory control in monkeys and humans. J. Neurosci. 31, 5181-5183.

Zandbelt, B. B., and Vink, M. (2010). On the role of the striatum in response inhibition. PLOS ONE 5, e13848. doi:10.1371/journal.pone.0013848

Conflict of Interest Statement: The authors declare that the research was conducted in the absence of any commercial or financial relationships that could be construed as a potential conflict of interest.

Received: 28 November 2011; accepted: 14 February 2012; published online: 05 March 2012.

Citation: Criaud M, Wardak C, Ben Hamed S, Ballanger $B$ and Boulinguez $P$ (2012) Proactive inhibitory control of response as the default state of executive control. Front. Psychology 3:59. doi: 10.3389/fpsyg.2012.00059

This article was submitted to Frontiers in Cognitive Science, a specialty of Frontiers in Psychology.

Copyright (C) 2012 Criaud, Wardak, Ben Hamed, Ballanger and Boulinguez. This is an open-access article distributed under the terms of the Creative Commons Attribution Non Commercial License, which permits non-commercial use, distribution, and reproduction in other forums, provided the original authors and source are credited. 